\title{
Fluorescence In Situ Hybridization
}

National Cancer Institute ( $\mathrm{NCl})$

\section{Source}

National Cancer Institute (NCI). Fluorescence in situ hybridization.

A technique used to identify the presence of specific chromosomes or chromosomal regions through hybridization (attachment) of fluorescently-labeled DNA probes to denatured chromosomal DNA. Examination through a microscope under fluorescent lighting detects the presence of the colored hybridized signal (and hence presence of the chromosome material) or absence of the hybridized signal (and hence absence of the chromosome material). Also called FISH. 